\title{
Singulière cohérence
}

\author{
Judith Wulf \\ Université Rennes 2 \\ j.wulf@free.fr
}

\section{Introduction}

Envisagée dans sa dimension interprétative, la cohérence pose le problème de l'identification des règles et des contraintes qui la régissent. La linguistique textuelle met notamment au jour des difficultés reposant sur le fait qu'il s'agit d'articuler divers types de contraintes, marqueurs d'intégration linéaire mais également surdétermination globale, comme le précisent Jean-Michel Adam et Françoise Revaz (1989). Certains outils, connecteurs ou anaphoriques, sont ainsi dédiés à la cohésion du texte; d'autres, «embrayeurs de points de vue, organisateurs textuels renégociant le linéaire, progression thématique et continuité référentielle, thématisation et isotopies fonctionnent comme des signaux qui instaurent un mode de réception », comme le rappelle Anna Jaubert (2006: 19). Dans une perspective stylistique, nous nous intéresserons au statut particulier d'outils qui entrent en jeu dans la cohérence de manière singulière, non au sens où il s'agirait de traits idiolectaux mais parce que leur fonctionnement dépend d'un contexte donné. Nous verrons qu'ils correspondent à des normes d'un type particulier et entrent dans une relation de configuration réciproque avec le contexte, ce qui leur confère certaines propriétés.

\section{La question du singulier}

L'un des principaux problèmes d'évaluation de la cohérence, c'est qu'elle engage la description d'une situation de communication concrète dans ce qu'elle a de plus particulier: on peut faire une typologie des outils de cohésion, mais il s'avère plus délicat de faire l'inventaire des marqueurs de cohérence, dans la mesure où le caractère concret de la situation de communication y est primordial. Une telle approche découle en fait d'une logique du signe envisageant le signifiant à la fois comme donné et comme point de départ de l'interprétation. Or l'observation des textes dits littéraires révèle un fonctionnement un peu différent: nombreux sont les outils d'organisation qu'on peut qualifier d'endogène dans la mesure où ils ne sont pertinents que dans un contexte donné ; ils ne seront pas employés de la même manière et ne joueront pas le même rôle ou ne produiront pas les mêmes effets dans un autre contexte. Loin du principe qui veut qu'à tel outil grammatical corresponde telle fonction prédéterminée, le texte littéraire privilégie une logique interprétative contextuelle selon laquelle à telle configuration spécifique s'attache une interprétation complexe particulière $^{1}$. Dans cette perspective, on ne peut penser simplement l'action du contexte en termes de modification, de déformation ou d'écart mais bien de configuration des unités pertinentes, dans la mesure où celles-ci sont identifiées dans l'interprétation (Neveu, 2007).

Un second problème concerne le cadre de traitement du singulier. Chaque genre de discours codifie ses propres contraintes et plus l'échange est régulé, plus il est facilité. La plupart des situations de discours tendent donc à réduire l'occurrence singulière pour mieux la maitriser. Ce n'est pas le cas du régime littéraire qui aura au contraire tendance à le valoriser comme partie intégrante de l'art créateur qui le constitue. Cela ne signifie pas que tel ou tel régime discursif contient plus ou moins de singulier, mais qu'il est peut-être plus facile à observer dans telle ou telle situation. De ce fait chaque cas singulier nous renseigne sur des cas de figure possibles, le texte littéraire proposant des exemples attestés dans une version maîtrisée qui leur confère une certaine lisibilité. 
Ce problème se double du caractère fondamentalement interactionnel de la cohérence : comme le rappelle Marie-Paule Pery-Woodley (2001), la «notion de cohérence réunit en fait deux facettes distinctes du fonctionnement des textes : la construction par le(s) scripteur(s) d'un discours cohérent dont le texte est la trace ; l'élaboration par le lecteur, à partir de cette trace, d'un modèle interprétatif cohérent. ». L'idée de singularité se double donc immédiatement ici d'un problème de multiplicité, qui suggère qu'en matière de cohérence seule existe les singularités au pluriel. On insistera donc sur le fait que le singulier se distingue de l'individuel. Contrairement à certaines recherches actuelles sur l'idiolecte (Neveu, 2005), nous n'assimilerons pas le singulier compris comme phénomène unique et l'idée qu'il ne concerne qu'un seul énonciateur. Par opposition, on envisagera le singulier comme événement linguistique se développant à partir d'un espace de partage qu'il configure à son tour en mettant en perspective les possibles de la langue. Le singulier n'est pas lié à un sujet existentiel mais à un jeu de déplacements. En ce sens, il est préindividuel, en voie de discrétisation et de stabilisation.

M'inscrivant dans une perspective stylistique ${ }^{2}, \mathrm{j}^{\prime}$ insisterai donc sur les effets de cohérence à réception : ce qui est singulier ce n'est pas seulement telle ou telle énonciation, ce sont ses conditions d'existence concrètes, prenant en compte la question des effets. Pris en ce sens, les phénomènes singuliers possèdent deux qualités principales, être «transitionnel» et «potentiel » ${ }^{3}$. On peut en effet tout d'abord les qualifier de transitionnels dans la mesure où ils opèrent dans un espace intermédiaire ou se chevauchent deux aires, celles du producteur et celle du récepteur de l'énoncé, celle du texte et celle du monde, celle de la norme et celle de la création. Le singulier n'appartient donc ni au locuteur comme idiolecte, ni au récepteur ni à la langue : il s'agit d'un espace de partage d'ordre interprétatif qui n'est ni commun ni individuel et qui n'est pas exempt d'oppositions, ce qui le rend paradoxal : comment si producteur et récepteur ne s'entendent pas sur des normes communes peuvent-ils se rencontrer? Comment un phénomène non institué peut-il fonctionner comme norme ? C'est que cet espace transitionnel, est également un espace de valorisation problématique. Le principal apport du singulier n'est pas en effet qu'il est caractéristique ou qu'il se distingue, mais qu'il fait signe vers un possible de la langue. Si le texte intéresse, c'est probablement parce qu'il parvient à poser une question qui regarde le lecteur. Ce faisant, il pointe des possibilités qui se réaliseront à réception. Un texte n'est donc pas incohérent en soi mais pour tel ou tel lecteur, ce qui implique que sa cohérence dépend de conditions concrètes de réalisation et pas seulement de critères structuraux. En ce sens il n'y pas actualisation mais (re)création cohérente.

\section{Statut textuel}

Dans cette perspective, on peut distinguer trois modes de fonctionnements des dispositifs entrant dans le mécanisme de cohérence: les outils dédiés qui, comme les connecteurs véhiculent des «instructions interprétatives ${ }^{4} »$; les systèmes hétérogènes qui relèvent à la fois de la phrase et du discours. Reposant en particulier sur l'ordre des mots, sur leur disposition, leur répétition ou leur absence, ils se caractérisent par une absence d'autonomie locale qui met en jeu le niveau global de l'énoncé. A propos des constructions détachées, par exemple, Bernard Combettes souligne le «conflit» qui s'établit bien souvent «entre contraintes discursives et contraintes syntaxiques » $(1996: 85)$. C'est le cas également pour l'ellipse qui est décrite en fonction de critères syntaxiques tout en étant un « puissant facteur de cohésion » (Charolles, 1995 : $127)^{5}$.

On distinguera un troisième cas dans lequel les marques relationnelles sont configurées de manière stylistique, c'est-à-dire que leur identification dépend de critères non pas structuraux mais perceptifs. Dans ces conditions, ellipse ou détachement ne sont pas perçus comme écart par rapport à une norme qui instituerait la phrase complète et liée comme première, mais comme manque ou détachement perçus comme effets saillants ${ }^{6}$. Dans cette perspective, le comportement d'un constituant dans la phrase n'est pas suffisant pour le singulariser; il faut qu'il y ait mise en scène ${ }^{7}$. 
Ce phénomène est très net dans le cas des constructions détachées. En les envisageant dans une perspective stylistique, on constate que la question n'est pas de savoir quelles sont les marques qui permettent de les définir a priori mais de quelle manière elles sont mises en scène en contexte et ce que cela apporte à la configuration du parcours interprétatif. Le détachement apparaît ainsi non comme un universel mais comme un principe variationnel qui va du non perçu à la mise en relief mais également d'une légère discontinuité à la mise en avant de la rupture, en passant par toutes les formes de décrochage.

Du coup, la mise en question la plus spectaculaire du détachement repose sur sa mise en question. C'est le cas des prédicats autonomes qui présentent des «similitudes» avec l'apposition, d'où résulte une figure d'hyperbate (Fromilhague, 2007).

L'encaissement du chemin creux était comble de chevaux et de cavaliers inextricablement amoncelés. Enchevêtrement terrible. Il n' y avait plus de talus. (Hugo, Les Misérables)

Dans cet exemple, la présence d'un point censé interdire la rection, met en avant l'idée de rupture. Cet effet est encore plus net lorsque la typographie se joint à ponctuation :

Le vent, c'est-à-dire cette populace de titans que nous appelons les souffles. L'immense canaille de l'ombre. (Hugo, Les Travailleurs de la mer)

D'un côté le segment, «l'immense canaille de l'ombre » apparaît comme le prolongement du mouvement de reformulation clairement modalisé par le connecteur «c'est-à-dire », mais de l'autre il est isolé sur une ligne paragraphe. C'est donc de manière contrastive que le détachement se fait ici sentir. Ce procédé est également fréquent chez Michelet :

Elle passait les jours au lit; lui à ses pieds, agenouillé sur des coussins comme on faisait alors.

Spectacle intéressant ! ( Michelet Richelieu et la Fronde)

Ce type de procédé n'est pas réservé aux détachements à droite : «Phénomène terrible ! Chez le cardinal même et devant lui, le succès fut complet » (Michelet, Richelieu et la Fronde). Dans cet exemple, il s'agit d'une variante d'un processus de détachement systématique qui participe à la constitution d'une véritable matrice rythmique, comme nous le verrons plus bas. Assez discret lorsqu'il est pris de manière isolée, le détachement devient évident par sa répétition à l'échelle de l'ouvrage.

Selon la stratégie expressive, le détachement peut ainsi être préparé plus ou moins progressivement. Un auteur comme Hugo, par exemple, a l'habitude de s'appuyer sur les phrases les plus simples ou les détachements les plus scolaires pour mieux rendre saillant certains segments :

Le doyen, qui était le révérend Jaquemin Hérode, était assis ; l'évangéliste et le registraire étaient debout. Le livre, ouvert, était sur la table. à côté, sur une crédence, s'étalait un autre livre, le registre de paroisse, ouvert également, et sur lequel un oeil attentif eût pu remarquer une page fraîchement écrite et dont l'encre n'était pas encore séchée. Une plume et une écritoire étaient à côté du registre. (Hugo, Les Travailleurs de la mer)

Dans cet exemple, on peut comparer les premières phrases, dans lesquelles la construction détachée en relation directe avec le substantif auquel elle se rapporte est à peine sensible comme telle, et les phrases suivantes qui la mettent en scène par une hyperbate en escalier. Par contraste avec les phrases équilibrées du début, les phrases suivantes donnent l'impression de se prolonger au-delà de leur limite rythmique. Cette mise en scène est renforcée par la présence de la dernière phrase qui revient à une structure simple, arrêtant ainsi le mouvement contrastif initié par les premières. Ainsi encadrée, la mise en figure du détachement est plus sensible. 
Balzac au contraire a plus souvent tendance à s'appuyer sur une saillance par accumulation. On comparera par exemple un segment du type : «Vulcain, avec sa laideur et sa force, n'est-il pas l'emblème de cette laide et forte nation » et les constructions détachées qui s'accumulent dans la suite de la phrase :

Vulcain, avec sa laideur et sa force, n'est-il pas l'emblème de cette laide et forte nation,
sublime d'intelligence mécanique, patiente à ses heures, terrible un jour par siècle,
inflammable comme la poudre, et préparée à l'incendie révolutionnaire par l'eau-de-vie,
enfin assez spirituelle pour prendre feu sur un mot captieux qui signifie toujours pour elle :
or et plaisir! (Balzac, La Fille aux yeux d'or)

C'est encore plus net lorsque l'accumulation se situe en incise, créant une disjonction qui sollicite de manière importante l'attention du lecteur: pour que ce type de détachement soit perçu comme tel, il est nécessaire qu'il se combine avec un principe d'accumulation

(...) dans une de ces joyeuses journées donc, un jeune homme, beau comme était le jour de ce jour-là, mis avec goût, aisé dans ses manières (disons le secret) un enfant de l'amour, le fils naturel de lord Dudley et de la célèbre marquise de Vordac, se promenait dans la grande allée des Tuileries. (Balzac, La Fille aux yeux d'or)

Enfin, de manière plus simple, le décrochage peut également être exhibé sous forme de parenthèses :

(...) ceux qui ont avorté dans l'escalade et le bris de clôture (car les grands faiseurs d'effractions planent et restent en haut), les ouvriers et les ouvrières du mal, les drôles et les drôlesses. (Hugo, Les Travailleurs de la mer)

Important dans le cas du détachement, ce mode de configuration contextuelle est encore plus net dans le cas de l'ellipse : dépourvue de signifiant, son identification ne peut en effet que reposer que sur des marqueurs de saillance indirecte orchestrés en contexte, ce qui pose à nouveau la question de l'autonomie locale.

L'un des procédés les plus courants consiste à abréger une structure instanciée préalablement en paradigme par sa répétition :

Cette ambition introduit la pensée dans la seconde des sphères parisiennes. Montez donc un étage et allez à l'entresol ; ou descendez du grenier et restez au quatrième ; enfin, pénétrez dans le monde qui a quelque chose : là, même résultat. (Balzac, La Fille aux yeux d'or)

Dans cet extrait de La Fille aux yeux d'or, la répétition d'une construction similaire, centrée autour d'une injonction, institue de manière implicite un paradigme cotextuel sur lequel vient se détacher le dernier segment phrastique ${ }^{8}$. C'est la brusque rupture du paradigme qui fait ressentir l'abrègement.

Structure abrégée et structure complète sont donc des notions relatives qui se configurent mutuellement en contexte comme effet. Les faire s'enchaîner permet de les mettre en valeur de manière contrastive comme c'est le cas dans la fameuse métaphore du miroir dans Le Rouge et le Noir :

Eh, monsieur, un roman est un miroir qui se promène sur une grande route. Tantôt il reflète à vos yeux l'azur des cieux, tantôt la fange des bourbiers de la route. Et l'homme qui porte le miroir dans sa hotte sera par vous accusé d'être immoral! Son miroir montre la fange, et vous accusez le miroir ! (Le Rouge et le noir)

Dans cet exemple, la conjonction de coordination «Et » placée en début de phrase signale qu'une première partie est sous-entendue. Mais seule la reformulation, dans la phrase qui suit, du segment sous la forme d'une structure logique complète («son miroir montre la fange, et vous accusez le miroir») produit rétrospectivement l'effet de place vide.

Parfois c'est la structure abrégée qui est récurrente et la structure complète que met en relief sa rareté. Ce type d'exemple correspond moins à une logique de rupture que de réciprocité : 
Dans cet exemple de monologue intérieur, se succèdent différentes zones d'abrègement ${ }^{9}$ de la structure la plus complète qu'exemplifie le segment «je vais seul, le long des maisons, silencieusement». Des zones dans lesquelles procès verbal ou énonciateur sont effacés laissent ainsi la place à d'autres dans lesquelles c'est le repère spatial ou le thème de la description qui disparait. Malgré le caractère très compact du texte, le choix d'une ponctuation plus légère et l'absence de cadratif, on peut distinguer différentes zones ou séquences selon que se répète tel ou tel type d'abrègement.

Le jeu de l'anaphore peut également contribuer à souligner l'effet d'effacement d'un élément de la chaîne de référence :

Qui sait? peut-être avons-nous encore des sensations après notre mort, disait-il un jour à Fouqué. J'aimerais assez à reposer, puisque reposer est le mot, dans cette petite grotte de la grande montagne qui domine verrières. Plusieurs fois, je te l'ai conté, retiré la nuit dans cette grotte, et ma vue plongeant au loin sur les plus riches provinces de France, l'ambition a enflammé mon cœur : alors c'était ma passion... Enfin, cette grotte m'est chère et l'on ne peut disconvenir qu'elle ne soit située d'une façon à faire envie à l'âme d'un philosophe... Eh bien ! ces bons congréganistes de Besançon font argent de tout ; si tu sais t’y prendre, ils te vendront ma dépouille mortelle...

Fouqué réussit dans cette triste négociation. Il passait la nuit seul dans sa chambre, auprès $\mathrm{du}$ corps de son ami, lorsqu'à sa grande surprise, il vit entrer Mathilde. (Stendhal, Le Rouge et le noir).

Dans cette séquence qui raconte la mort de Julien à la fin du Rouge et le noir, deux types d'ellipse se combinent: les points de suspension signalent une ellipse narrative tandis que l'anaphore «le corps de son ami » qui renvoie au référent «Julien » repris par «il» dans tout le premier paragraphe, indique, dans le second, l'effacement de l'étape principale de l'évolution référentielle ${ }^{10}$.

Comme on le constate, ellipse et détachement présentent plusieurs similitudes. Notés par les linguistes, ces points communs sont également exploités par les écrivains qui n'hésitent pas à faire converger leurs effets :

De temps en temps on rencontrait des traces de campements, des places brûlées, des herbes foulées, des bâtons en croix, des branches sanglantes. Là on avait fait la soupe, là on avait dit la messe, là on avait pansé des blessés. Mais ceux qui avaient passé avaient disparu. Où étaient-ils ? bien loin peut-être. Peut-être là tout près, cachés, l'espingole au poing. Le bois semblait désert. Le bataillon redoublait de prudence. Solitude, donc défiance. On ne voyait personne ; raison de plus pour redouter quelqu'un. On avait affaire à une forêt mal famée. (Hugo, Quatrevingt-Treize)

Comme tous les dispositifs discursifs, ellipse et détachement entrent avec le contexte dans un rapport réciproque de configuration. Mais ils joueront un rôle d'autant plus complexe dans la formation du parcours interprétatif qu'ils auront reposé sur des mécanismes perceptifs singuliers de type stylistiques.

\section{Configuration contextuelle}

La particularité de ces outils singuliers c'est qu'une fois mis en scène en contexte, ils le configurent à leur tour, instaurant un processus d'articulation réciproque entre local et global. Ce type de fonctionnement, qui joue un rôle important dans tout type de discours, est particulièrement représenté dans le texte littéraire, qui 
l'utilise comme impulsion : il s'agit de provoquer un sentiment de manque ou un effet d'incomplétude obligeant le lecteur à quitter le niveau local et à s'ouvrir à un niveau plus large. Configurées par le contexte, ces constructions elliptiques ou détachées entretiennent avec lui un rapport de réciprocité qui vient renforcer l'ambiguïté de leur statut entre rupture et lien, autonomie et dépendance. Par ailleurs leur identification comme configuration stylistique a des conséquences pragmatiques ${ }^{11}$.

\subsection{Configuration thématique}

En ce qui concerne les constructions détachées, elles ont un rôle thématique qui consiste à maintenir un référent saillant dans la mémoire du récepteur ou à préparer sa disponibilité (Combettes 1998). Quant à l'ellipse, Michèle Bigot (2008) à la suite de Lucien Cherchi (1978), insiste sur son rôle de cohésion, mais également de gestion de l'information et d'articulation des plans textuels. Contrairement au fonctionnement endophrastique de l'ellipse, la configuration contextuelle invite le lecteur à récupérer l'élément manquant à un niveau transphrastique.

Ellipse et constructions détachées fonctionnent donc d'abord comme opérateurs de liaison entre différents segments du texte. Mais il s'agit d'un lien qui possède des propriétés spécifiques. La première propriété de ce lien est qu'il est nécessaire.

C'est particulièrement net pour l'ellipse. Dans la métaphore stendhalienne du miroir citée plus haut, on peut ainsi distinguer deux types d'articulation : la structure complète, autonome, permet au lecteur d'en rester à un niveau phrastique tandis que la structure elliptique rend nécessaire une mise en relation avec la séquence textuelle. Ce type de construction n'est pas neutre d'un point de vue pragmatique, puisque c'est sur lui que repose l'ironie du passage.

On retrouve un dispositif similaire avec les constructions détachées qui, dans beaucoup de cas, assurent une continuité thématique avec le contexte antérieur tout en servant de cadre à un énoncé postérieur (Combettes, 1998). Paradoxal, ce lien qui repose sur un détachement possède des propriétés spécifiques.

Le vent, c'est-à-dire cette populace de titans que nous appelons les souffles.

L'immense canaille de l'ombre. (Hugo, Les Travailleurs de la mer)

Dans cet exemple, le détachement, constitue une transition entre la section II, III, 1 du roman, à valeur documentaire et la section II, III, 2 à portée métaphysique.

Ces constructions ne servent donc pas seulement de lien, elles orientent également la lecture selon un parcours qui ne se limite pas au simple enchaînement linéaire de gauche à droite :

La nature vient au secours de tous les abandons; là où tout manque, elle redonne tout entière ; elle refleurit et reverdit sur tous les écroulements ; elle a le lierre pour les pierres et l'amour pour les hommes.

Générosité profonde de l'ombre. (Hugo, L'Homme qui rit)

Placé en fin de chapitre, ce segment détaché se rattache au paragraphe qui précède mais ouvre également sur l'ensemble du chapitre pour lequel elle fait office de conclusion. Elle fait notamment écho aux réflexions sur la destinée complexe de Gwynplaine («produit d'une fatalité compliquée d'une providence», «point d'intersection des deux rayons d'en bas et d'en haut, du rayon noir et du rayon blanc »), en même temps qu'elle exemplifie par les propriétés de ce détachement le principe atomique qui explique cette cohérence paradoxale.

Le détachement correspond généralement à un mouvement de remontée en amont. Mais dans le segment détaché se situe au milieu d'un paragraphe (et ne constitue pas le paragraphe à lui tout seul), la polarisation s'estompe : 
L'ombre est un silence (...) Les syllogismes, les querelles, les négations, les systèmes, les religions, passent dessus sans la diminuer. Cette notion, l'ombre tout entière l'affirme. Mais le trouble est sur tout le reste. Immanence formidable. L'inexprimable entente des forces se manifeste par le maintien de toute cette obscurité en équilibre. (Hugo, Les Travailleurs de la mer)

Ici, le segment «Immanence formidable » résume de manière conclusive les remarques en amont tout en relançant l'attention du lecteur vers l'aval du texte.

Cette propriété est également valable pour les segments textuels comprenant des ellipses forment une séquence avec une zone textuelle généralement placée en amont. C'est particulièrement net dans l'exemple suivant :

(...) ma tête était tombée... elle avait roulé, rebondi sur le hideux parvis de l'échafaud, et, prête à descendre toute meurtrie entre les mains des enfants, des jolis enfants de Larisse, qui se jouent avec des têtes de morts, elle s'était rattachée à une planche saillante en la mordant avec ces dents de fer que la rage prête à l'agonie. De là je tournais mes yeux vers l'assemblée, qui se retirait silencieuse, mais satisfaite. Un homme venait de mourir devant le peuple. Tout s'écoula en exprimant un sentiment d'admiration pour celui qui ne m'avait point manqué, et un sentiment d'horreur contre l'assassin de Polémon et de la belle Myrthé. - Myrthé ! Myrthé ! m'écriai-je en rugissant mais sans quitter la planche salutaire. - Lucius ! Lucius ! répondit-elle en sommeillant à demi, tu ne dormiras donc jamais tranquille quand tu as vidé une coupe de trop! (Nodier, Smarra ou les Démons de la nuit)

Dans le segment en caractères gras, l'information manquante suggérée par le connecteur argumentatif ne peut être récupérée qu'en raccordant la phrase avec celle qui, quelques lignes plus haut, indiquait que la tête du personnage « s'était rattachée à une planche saillante en la mordant avec ces dents de fer ». Ouvrant la lecture d'un point localisé à un segment plus large, ce dispositif crée un effet de porosité entre les deux plans du rêve et du réveil : non seulement l'ellipse invite le lecteur à remonter plusieurs lignes en amont pour configurer une séquence complète, mais elle le conduit à revoir la successivité textuelle des deux niveaux fictifs pour les percevoir sous l'angle d'une superposition.

Dans le cas de la zone elliptique formée par récurrence, il n'y a pas un point d'ancrage de la récupération qu'on pourrait localiser à gauche ou à droite. Chaque élément joue tour à tour le rôle de centre pour les autres selon un dispositif de montage et de diffusion. De ce fait le parcours interprétatif se fait en zigzag selon un mouvement de va-et-vient. C'est particulièrement net dans le cas de l'exemple de Dujardin. Ce principe empêche la représentation synthétique et donne l'impression d'être plongé au cœur du référent.

\subsection{Configuration interprétative}

Qu'il s'agisse d'ellipse ou de détachement mis en scène, l'effet de dépendance et les conditions de sa configuration ont donc une importance aussi bien discursive que pragmatique. En régime littéraire, ce genre de dispositif qu'on peut rencontrer dans n'importe quel énoncé, se complexifie fréquemment pour s'orienter avant tout vers une fonction plus spécifiquement interprétative. Sans cette nouvelle dimension, on ne pourrait parler de cohérence singulière. Contrairement à la plupart des énoncés standards qui fonctionnent sur le modèle de l'instruction $^{12}$, guidant, de manière assez directive, le lecteur dans son parcours interprétatif, le texte littéraire a tendance à le laisser participer de manière beaucoup plus libre. On en a un exemple lorsque le mouvement de dépendance initié par les organisateurs textuels singuliers ne trouve pas de point d'aboutissement. Il est des cas, en effet, où l'ellipse ne peut conduire à aucune récupération univoque, où le détachement n'est lié à aucun point précisément localisable. Au-delà du décrochage syntaxique permettant un lien thématique, existe un décrochage thématique permettant une impulsion interprétative. C'est le cas 
notamment pour les exemples pour lesquels l'enchaînement thématique est ambigu voire impossible à reconstituer :

Cette ambition introduit la pensée dans la seconde des sphères parisiennes. Montez donc un étage et allez à l'entresol ; ou descendez du grenier et restez au quatrième ; enfin, pénétrez dans le monde qui a quelque chose : là, même résultat. (Balzac, La Fille aux yeux d'or)

Dans cet exemple, déjà cité, l'attente déçue d'une instruction injonctive se double de la recherche sans résultat d'une récupération thématique. Répétition et variation se combinent pour ne laisser au lecteur d'autre choix que de construire lui-même le contenu d'une injonction qu'il ne peut déduire des précédentes. Ce mouvement est renforcé par l'indétermination de «là » qui, en raison de son indifférence au statut catégoriel du terme auquel il renvoie, n'a pas d'antécédent linguistique clairement circonscrit: doit-on l'interpréter comme reprenant «le monde», comme résumant les différents lieux, ou comme désignant une étape bilan dans la description?

Ce faisant, le narrateur implique le lecteur en l'intégrant au dispositif énonciatif :

Dans cet instant où la distance effaça l'ourque et où l'enfant ne vit plus rien, l'enfant s'en alla au nord pendant que le navire s'en allait au sud.

Tous s'enfonçant dans la nuit. (Hugo, L'Homme qui rit)

Détaché en fin de chapitre, ce segment reprenant les occupants de l'ourque et l'enfant, cités dans la phrase qui précède, ouvre sur un segment narratif plus large mais renvoie également à la situation d'énonciation en suggérant qu'on passe du simple exposé des faits à une étape énigmatique.

De manière générale, il s'agit d'instaurer une relation déhiérarchisée entre narrateur et lecteur: «Dans le *Lyse-*Fiord, presque toujours la lame est tranquille ; le ciel est serein ; lieu terrible. Où est le vent ? Pas en haut. Où est le tonnerre ? Pas dans le ciel (Hugo, Les Travailleurs de la mer). Effaçant toute subordination textuelle, dans la logique d'une forme déhiérarchisante, le mécanisme exemplifie un espace paradoxal qui n'est plus organisé en fonction des coordonnées habituelles.

\subsection{Configuration rythmique}

Les exemples qui précèdent ne doivent pas laisser penser que ces outils de configuration singuliers ne sauraient concerner qu'un segment textuel. Ils peuvent également fonctionner à l'échelle de l'ouvrage. C'est le cas notamment lorsqu'ils s'inscrivent dans une matrice rythmique.

C'est un procédé qu'on rencontre dans Richelieu et la Fronde de Michelet et qui est intimement lié à une réflexion originale sur l'histoire : chaque remarque y est en effet dédoublée en deux perspectives différentes voire hétérogènes. A la construction linéaire, se superpose alors un dispositif de lecture en va-et-vient qui s'instaure progressivement jusqu'à devenir automatique. Dans la plupart des exemples, une version synthétique reprend l'exposé analytique : «Protestant, il se convertit pour une riche dot, qu'il réalisa en fausse monnaie d'Autriche, et acheta pour rien des confiscations, puis des soldats, des régiments, des corps d'armée, des armées. L'avalanche allait grossissant. »

Selon les cas, la reformulation synthétique peut être mise en valeur par un connecteur comme «bref »:

De là des fortunes subites, des ruines aussi, de grosses pertes, des batailles financières, de morts et des suicides de gens qui survivaient, mais pauvres, non plus hommes, mais ombres, des millionnaires devenus facchini ; comme un carnaval éternel ; bref, une société mouvante. 
Comme on l'a vu plus haut, la figure d'hyperbate est fréquente: «Ne pouvant imiter l'éloquence de la possédée de Marseille, elles suppléaient par le cynisme. Spectacle hideux!»; Surtout lorsque le segment est extraposé sur une ligne paragraphe :

Elle passait les jours au lit; lui à ses pieds, agenouillé sur des coussins comme on faisait alors.

Spectacle intéressant!

Parfois la perspective synthétique précède son développement analytique :

Phénomène terrible! Chez le cardinal même et devant lui, le succès fut complet. Acteurs et spectateurs avaient pris l'âme du Cid. Personne n'avait plus peur de rien. Le ministre resta le vaincu de la pièce, aussi bien que don Sanche, l'amant dédaigné de Chimène

Ce mécanisme de redoublement des perspectives analytique et synthétique est systématisé dans Richelieu et la Fronde au point qu'il entraîne un mode d'organisation rythmique du sens qui, fondé sur le va-et-vient, concurrence la linéarité de la lecture. Il est soutenu pour cela en contexte par d'autres mécanismes qui jouent également sur le va-et-vient, comme le redoublement d'un élément de la phrase pour le présenter sous deux angles différents ou le redoublement d'un pôle fonctionnel pour rapprocher deux perspectives sémantiques hétérogènes. Dans certains cas, plusieurs niveaux de redoublement peuvent également se combiner. Le redoublement de perspective peut concerner un même référent: «Comme le vieux duc de Savoie, notre parent et ennemi » ou encore « Le grand vengeur des guerres, la peste, impartiale, était venue ensuite ». Mais il peut également porter sur le simple contenu notionnel : «ils quittèrent leur camp, un cimetière, pour attaquer l'autre cimetière, qui était la ville.» Plusieurs niveaux de redoublement peuvent également se combiner : «Par quelles prises avaient-elles ressaisi le roi ? Par la plus imprévue : une femme, un amour... Cet insensible, ce malade saigné à blanc, si pâle, qui faisait presque peur, on trouva l'art de le rendre amoureux. »

De nombreux phénomènes de redoublement (dont je n'ai reproduit ici que quelques exemples) ponctuent donc le texte de Michelet. Systématisés, ils créent dans le texte un véritable patron pragmatique qui guide implicitement le lecteur vers une double représentation de l'objet. Le schéma rythmique permet alors de pallier l'embarras conceptuel, de maintenir l'hétérogénéité sémantique tout en lui préservant une cohérence. La mise en texte obéit en effet à des lois qui ne sont pas forcément celles de l'histoire (telle qu'il propose de la penser) et qui risquent d'en fausser la représentation. La recherche d'une cohérence singulière correspond donc à un enjeu complexe : il s'agit de traduire pour le lecteur l'esprit du XVIIe siècle tout en partageant avec lui une réflexion épistémologique originale sur le nouveau paradigme historique qui se développe après la révolution française.

Ecrit après le succès de L'Oiseau qui propose notamment une réflexion sur le point de vue, et en pleine vogue du stéréoscope, il n'est pas fortuit que Richelieu et la Fronde semble mettre en avant un mode d'organisation de la représentation fondé non sur la décomposition linéaire une organisation en épaisseur. De même que, confronté à la vision simultanée de deux images différentes du même objet, notre cerveau interprète ce que voient nos yeux en une seule image en relief, l'esprit du lecteur, confronté à un redoublement saillanciel qu'il soit syntaxique et/ou sémantique traite le phénomène de manière globale. Lorsque ce mécanisme est systématisé, à l'automatisme du traitement linéaire de l'information se substitue un réflexe de saisie bipolaire. Si l'agencement des mots dans le texte reste linéaire, sa lecture, son interprétation et en somme son organisation effective ne l'est plus.

Le rythme apparaît ainsi comme un facteur de cohérence qui fait l'économie des outils de cohésion souvent figés par le cadre générique auquel appartient tel ou tel texte. Dans le cas de Michelet, il correspond à un aspect essentiel de la problématique historique, à savoir trouver un mode d'écriture qui ne trahisse pas la complexité du matériau historique. Contrairement à la forme schématique, la forme rythmique est à même de rendre compte d'éléments vivants, de manière vivante ${ }^{13}$. Nombreux sont ceux qui virent dans ce choix une 
trahison de la vérité historique au profit d'une virtuosité quelque peu parasite. Face aux critiques d'un SainteBeuve sur ce point, Michelet réaffirme la nécessité d'une forme qui puisse faire preuve d' " entrain ». Loin d'être secondaire, cette dimension stylistique est essentielle pour agencer "toute une masse de choses diverses » dans une dynamique qui puisse « donner le sentiment de la vie historique ${ }^{14}$.

\section{Bilan sur les propriétés et enjeux de cette cohérence}

On l'a vu, toute la difficulté d'étudier la cohérence, repose sur son caractère singulier de configuration contextuelle. Si cet aspect n'est pas propre au texte littéraire, celui-ci fournit un excellent espace d'observation, dans la mesure où il valorise de manière maîtrisée ce que d'autres régimes de discours cherchent à réguler. De ce fait, l'observation des conditions dont la singularité est prise en compte influence le fonctionnement de la cohérence et lui confère certaines propriétés.

L'analyse du discours en régime littéraire montre tout d'abord la part fondamentale de la dimension perceptive dans la structuration du texte, notamment pour guider l'interprétation du lecteur. Les mécanismes inférentiels, dans cette perspective ne sont pas uniquement de nature logique. On peut percevoir un rythme, la récurrence d'une forme, des aspects visuels ou d'autres types de phénomènes saillants sans pour autant en être conscient. Peuvent alors être pertinents des éléments qui n'ont pas été identifiés comme tels, ce qui ne signifie pas qu'ils ne sont pas intentionnels. C'est notamment ce qui permet de distinguer la dynamique esthétique du texte littéraire (mais qui ne lui est pas réservée) d'une simple sensation passive. Tout interprétant de cohérence est donc également une «esthésie» pour reprendre le terme de F. Rastier ${ }^{15}$, c'est-à-dire un schéma, une organisation cognitive. Simplement la dimension perceptive étant difficile à maîtriser, le régime courant de discours cherche à en faire l'économie là où le régime littéraire tentera de le valoriser en raison de son ouverture et de la rentabilité potentielle qu'il permet.

Elle permet également de préciser l'importance de l'influence des conditions d'émergence sur les opérateurs de cohérence : les opérateurs fonctionnent donc bien comme des signaux ou des déclencheurs ${ }^{16}$, mais de deuxième niveau. Il faut d'abord qu'ils aient été constitués en usage grâce à d'autres déclencheurs ou signaux. Cela entraîne un lien de réciprocité entre local et global entrant dans une relation de cohérence qui possède certaines propriétés.

La première propriété de cette relation est qu'elle ne repose pas sur un lien démarcatif. Contrairement aux connecteurs qui, tout en instaurant une liaison, marquent la frontière qui sépare les différents éléments qu'ils relient, les opérateurs singuliers insistent sur le lien au détriment de la rupture. Ce dispositif permet de pallier une segmentation trop restreinte pour engager le lecteur vers de plus vastes unités. La rentabilité stylistique de ce dispositif textuel est particulièrement nette pour l'ellipse dans le cas de Dujardin où elle favorise l'esthétique de continuité qui fonde le principe du monologue intérieur : on glisse d'une zone à une autre sans avoir à passer par le détour d'un marqueur. On retrouve un effet similaire pour le détachement, dans le cas de l'exemple des Misérables. Cette réflexion sur le statut des modalités du lien est également particulièrement sensible dans l'exemple de Quatrevingt-Treize cité plus haut : que la seule occurrence dans l'ouvrage d'un donc détaché soit placé en incipit souligne bien la volonté de guider d'emblée le lecteur vers une posture interprétative plus souple.

La relation de cohérence singulière possède également une certaine souplesse. Mettant en avant rupture et décrochage autant que dépendance, il contraint assez peu les niveaux d'interprétation. Aussi apparaît-elle comme non dogmatique comme dans l'exemple suivant :

Son entrée dans cette auréole avait eu lieu. Entrée irrévocable.

Il était là chez lui.

Chez lui sur son siège comme le roi sur le sien. (Hugo, L'Homme qui rit) 
Effaçant toute subordination textuelle, dans la logique d'une forme déhiérarchisante, le mécanisme exemplifie la lutte contre la subordination politique.

La dernière des propriétés de l'outil singulier de cohérence est donc son caractère plastique qui permet d'articuler des modes sémiotiques hétérogènes. C'est le cas du rythme dans Richelieu et la Fronde, qui est à la fois expression mimétique de l'ambiguïté qui caractérise pour Michelet le XVIIe siècle, réflexion épistémologique originale sur l'histoire, exemplification sémiotique d'une mise en forme non schématique du devenir et opérateur de configuration stéréoscopique du texte.

Les exemples étudiés dans cet article permettent donc de mesurer l'importance de l'influence sur l'interprétation des conditions de configuration singulière des organisateurs discursifs. Rien n'est neutre en régime littéraire - qu'il s'agisse ou non d'un texte de fiction - et chaque choix déploie ses possibles interprétatifs sans les contraindre de manière prédictible. N'étant pas dédié à cette tâche, les organisateurs discursifs configurés en contexte apparaissent ainsi comme autant d'interprétants de cohérence qui peuvent ou non être investis à réception, visant ce qu'on pourrait qualifier de cohérance en acte.

\section{Références bibliographiques}

Achard-Bayle, G. (1998), «Référence, identité, changement », L'Information grammaticale, n77, p.50-53.

Adam, J.-M. et Revaz, F. (1985), « Aspects de la structuration du texte descriptif. Les Marqueurs d'énumération et de reformulation », Langue française $\mathrm{n}^{\circ} 81$, p. 59-98.

Bernard, C (1966), Le Passé recomposé : le roman historique français du XIXe siècle, Paris, Hachette.

Bigot, M. (2008), « Effacement et ellipse, du schème phrastique à l'organisation discursive » in Bigot, M. et Pitavy, J.-C., Ellipse et effacement, Saint-Etienne, PUSE.

Bonhomme, M (2006), «Entre grammaire et rhétorique : l'hyperbate comme extraposition problématique » in actes du colloque Les linguistiques du détachement, à paraitre.

Charolles, M. (1995), « Cohésion, cohérence et pertinence du discours », Travaux de linguistique, n² 29, p. 125-151.

Cherchi, L. (1978), « L’ellipse comme facteur de cohérence », Langue française, $\mathrm{n}^{\circ} 38$.

Combettes, B. (1996), «Facteurs textuels et facteurs sémantiques dans la problématique de l'ordre des mots : le cas des constructions détachées », Langue Française n¹11, p. 83-96.

Combettes, B. (1998), Les constructions détachées en français, Paris, Ophrys.

Cornish, F. (1990), «Anaphore pragmatique, référence et modèles du discours » in Kleiber et Tyvaert, L'anaphore et ses domaines, Paris Klincksieck.

Dujardin, E. (1931), Le Monologue intérieur, Messein.

Fromilhague, C. (2007), «Les constructions détachées, du simple, à l'exprès trop simple dans Fêtes galantes et Romances sans paroles », in Reggiani, C. et Stolz, C. Styles, Genres, Auteurs, Paris, PUPS, p. 95-109.

Gérard, C. et Wulf, J. (2008), «Le singulier dans les textes» in Bougault, L. et Wulf, J., Questions de stylistique \& Stylistiques en question, à paraître.

Halliday, M. et Hasan, R. (1976), Cohesion in English, Londres, Longman.

Jaubert, A. (2005), «Cohésion et cohérence : étapes et relais pour l'interprétation », in. A. Jaubert (dir.), Cohésion et cohérence. Etude de linguistiques textuelle, Lyon, ENS éditions. 
Jaubert, A. (2006), «Les ordres du discours en perspective, cohérence et pertinence », in Calas, F., Cohérence et discours, Paris, PUPS, p.15-21.

Lundquist, L (1994), La Cohérence textuelle, Fredericksberg, Samfundslitteratur.

Molinié, G. (2008), «Pratique et herméneutique », in Bougault, L. et Wulf, J., Questions de stylistique \& Stylistiques en question, à paraître.

Neveu, F dir. (2005), L'Idiolecte. Du singulier dans le langage, Cahiers de praxématique, ${ }^{\circ} 44$.

Neveu, F. (2007), «L'homonymie textuelle à l'épreuve de l'évocation - Quelques éléments de réflexion épistémologique pour une sémantique de l'interprétation » in Lagorgette (dir), Littérature et linguistique : diachronie / synchronie Autour des travaux de Michèle Perret, Chambéry.

Péry-Woodley, M-P. (2001), « Modes d'organisation et de signalisation dans des textes procéduraux », Langages, 141, p. 28-46.

Petitier, P. (2006), Michelet, L'homme histoire, Paris, Grasset.

Rastier, F. (1996), «Problématiques du signe et du texte », Intellectica, 23.

Rastier F. (2007), «Conditions d'une linguistique des normes», in G. Siouffi, A. Steuckardt (dir.) Les linguistes et la norme, Aspects normatifs du discours linguistique, Sciences pour la Communication, 81, Bern, Berlin, Bruxelles, Frankfurt am Main, New York, Oxford, Wien

Wulf, J. (2006), «Contribution stylistique à l'analyse de la notion de cohérence », in Calas, F. Cohérence et discours, Paris, PUPS, p.287-296.

Wulf, J. (2007), «Linguistique et singularité du texte littéraire », Littérature et linguistique : diachronie / synchronie - Autour des travaux de Michèle Perret, D. Lagorgette et M. Lignereux eds., CD-Rom, Chambéry, Université de Savoiep.552-563.

Wulf, J. (2008), «Ellipse et configuration stylistique du parcours interprétatif », in Bigot, M. et Pitavy, J.-C., Ellipse et effacement, Saint-Etienne, PUSE, p.21-280.

${ }^{1}$ C'est ce que décrit l'approche herméneutique du contexte, montrant que le signifiant doit lui-même faire l'objet d'une identification, laquelle " est précédée par les attentes et présomptions définies par le contrat propre au genre textuel». Loin d'en être à l'origine, celle-ci intervient à l'issue d'un processus interprétatif dans lequel le sens se construit selon des parcours qui vont « de signifié en signifié, aussi bien que du signifié vers le signifiant» (F. Rastier, $1996: 36$ et 35).

${ }^{2}$ Non pas au sens où nous rechercherons des caractères stylistiques propre à tel ou tel auteur mais dans l'idée qu'un certain nombre d'événements linguistiques se produisent comme effet, à réception pour reprendre l'opposition rappelée par G. Molinié (2008).

${ }^{3}$ Pour plus de précisions, voir l'article de C. Gérard et J. Wulf (2008).

${ }^{4}$ « invitant le lecteur à accomplir un certain nombre d'opérations inférentielles à partir du donné linguistique en cours de traitement et du contexte dans lequel ce donné apparaît. » (Charolles, $1995: 126)$

${ }^{5}$ Sur ce point voir M. Hallyday et R. Hasan (1976), L. Cherchi (1978), M. Bigot (2005).

${ }^{6}$ Développé pour l'ellipse dans J. Wulf (2008).

${ }^{7}$ Nous verrons comment ces éléments, qui tirent leur configuration singulière du contexte, participent à leur tour à l'organisation de ce dernier dans un rapport réciproque entre local et global.

${ }^{8}$ On le comprend, la question n'est pas ici de savoir si la phrase nominale est en soi une phrase elliptique mais de constater que l'absence de support verbal est de fait mise en scène dans un contexte qui l'institue comme place vide et, partant, comme prédicat non autonome. Ce phénomène est d'autant plus sensible si on le rapporte à la récurrence des injonctions verbales dans le reste du texte (voyez, examinez, constatez etc.). 


\footnotetext{
${ }^{9}$ Selon la définition que l'auteur lui-même propose du monologue intérieur : « Discours sans auditeur et non prononcé par lequel un personnage exprime sa pensée la plus intime, la plus proche de l'inconscient, antérieurement à toute organisation logique, c'est-à-dire en son état naissant, par le moyen de phrases réduites au minimum syntaxial, de façon à donner l'impression du tout venant » (1931:59).

${ }^{10}$ Comme le signale G. Achard-Bayle (1998: 51), «parmi les expressions descriptives, la définie est mieux à même de recatégoriser le référent ».

${ }^{11}$ C'est le cas notamment de l'hyperbate dont la perception comme figure modifie la portée pragmatique, comme l'explique Marc Bonhomme (2006).

12 «les expressions linguistiques servent d'instructions au lecteur pour utiliser ses connaissances du monde de manière spécifique en vue de construire une représentation cohérente du texte en question » (L. Lundquist, 1994, Préface de la deuxième édition, II).

${ }^{13}$ En cela, Michelet serait selon C. Bernard (1966) le représentant d'une histoire « résurrection » par opposition à une histoire « dissection » : «Au XIXe, ces tendances polarisent sans préjudice de fréquentes interférences, l'une une certaine Histoire romantique souvent accusée de virer au romanesque, et le roman historique lui-même : l'autre l'Histoire à visée scientifique. Cette dernière évalue les débris et compte des cadavres. » On peut même imaginer des cas de détournements rhétoriques dans lesquels une structure perceptive suffisamment suggestive masque un glissement sémantique, voire une rupture logique.

${ }^{14}$ Cité par P. Petitier (2006 : 132).

${ }^{15}$ F. Rastier (2001), « glossaire index des notions ».

${ }^{16}$ Pour reprendre l'expression de F. Cornish (1990).
} 